\title{
Tumor Findings Anatomical Region of Specimen
}

National Cancer Institute

\section{Source}

National Cancer Institute. Tumor Findings Anatomical Region of Specimen. NCI

Thesaurus. Code C119923.

The specific anatomical or biological region within the tissue or organ specimen used in the tumor findings assessment. 